\title{
Himalayites treubi DOUVILLE (1912), Fósil índice de Titoniano Superior en el sur- oeste del lago Titicaca, Puno; implicaciones bioestratigráficas.
}

\section{Himalayites treubi DOUVILLE (1912), Upper Titonian index fossil from southwestern Lake Titicaca, Puno; biostratigraphic implications.}

DOI: $10.46932 / \mathrm{sfjdv2n3-029}$

Received in: May 1st, 2021

Accepted in: Jun 30th, 2021

\author{
Newton Machaca \\ UNA-PUNO. Facultad de Ingeniería Geológica y Metalúrgica. Jr. Jorge Basadre 640 C.U. Puno. \\ Carlos Esqivel \\ Universidad Autónoma Del Estado De Hidalgo. Centro de Investigaciones Biológicas, Colonia \\ Carboneras, C.P.42184, Pachuca de Soto, Hidalgo, México. \\ E-mail: vmachaca@gmail.com \\ Sofía Benavent \\ Universidad Autónoma Del Estado De Hidalgo. Centro de Investigaciones Biológicas, Colonia \\ Carboneras, C.P.42184, Pachuca de Soto, Hidalgo, México. \\ E-mail: vmachaca@gmail.com
}

\section{RESUMEN}

Con motivo de la actualización de la geología regional del Cuadrángulo de Puno (32v) a escala al 50,000, se han desarrollado diversas labores de campo, como ésta contribución que documenta nuevos resultados de rocas mesozoicas del Grupo Yura en las inmediaciones del Distrito de Tiquillaca, (al SO del Lago Titicaca), tales afloramientos solían asignarse a rocas jurásicas del Grupo Lagunillas. El afloramiento en estudio se halla en el Cerro Yana Apacheta, en cuya quebrada principal se elaboró una columna estratigráfica y el perfil correspondiente en el que se encontró el amonite Himalayites treubi DOUVILLE (1912), estableciendo una correlación con las formaciones Chachacumane y Labra. El taxón se describe con base en cinco ejemplares y sus parámetros estándares de tasa de enrollamiento y ornamentación del costillaje. Se considera que el hallazgo de amonites del Tithoniano en el Cuadrángulo 32v amplía y documenta la columna previamente reportada para la localidad y establece una clara correlación con sedimentos del sur de Asia (Indonesia).

Palabras clave: amonites, Himalayites, Tiquillaca, Puno, Perú

\begin{abstract}
On the occasion of the updating of the regional geology of the Puno Quadrangle (32v) at 50,000 scale, several field works have been developed, such as this contribution that documents new results of Mesozoic rocks of the Yura Group in the vicinity of the Tiquillaca District, (SW of Lake Titicaca), such outcrops used to be assigned to Jurassic rocks of the Lagunillas Group. The outcrop under study is located in Cerro Yana Apacheta, in whose main gorge a stratigraphic column and the corresponding profile was elaborated, in which the ammonite Himalayites treubi DOUVILLE (1912) was found, establishing a correlation with the Chachacumane and Labra formations. The taxon is described based on five specimens and its standard parameters of coiling rate and rib ornamentation. The finding of Tithonian ammonites in Quadrangle $32 \mathrm{v}$ is considered to extend and document the column previously reported for the locality and establishes a clear correlation with sediments from South Asia (Indonesia).
\end{abstract}


Key words: ammonites, Himalayites, Tiquillaca, Puno, Peru.

\section{INTRODUCCIÓN}

La presente investigación se desarrolló en el marco del Proyecto GR21 "Geología de los bordes de la cordillera Oriental y su relación con la Zona Subandina y Altiplano y con los Recursos Geológicos", de la Dirección de Geología Regional del Instituto Geológico Minero y Metalúrgico (INGEMMET); con la cátedra de geología de Campo II de la Facultad de Ingeniería Geológica y Metalúrgica de la Universidad Nacional de Altiplano (FIGIM-UNA-PUNO), con datos propios del proyecto, provenientes de la campaña de campo correspondiente al presente año, próximos a publicarse.

El presente estudio busca la caracterización de afloramientos con invertebrados fósiles en el Distrito de Tiquillaca al SO del Lago Titicaca, donde se ha producido históricamente información relevante, ya publicada, sobre evolución y características geológicas con la concurrencia de rocas y fósiles. Se conocen informes de campo y tesis de grado pero a nivel regional, los mismos que han servido como referencia para el presente estudio dentro de los cuales podemos señalar los estudios del PROYECTO INTEGRADO DEL SUR (Palacios et al, 1991), e informes de las cátedras de paleontología de la UNIVERSIDAD AUTÓNOMA DEL ESTADO DE HIDALGO, donde se tiene registros de la fauna ammonoidea y se han dado los primeros aportes en la cronología de las capas (i.e. Grupo Lagunillas, Jaillard \& Santander, 1992). Es objetivo de este trabajo dar a conocer las correlaciones y el material con el que se han considerado las descripciones taxonómicas; asimismo presentar a esta fauna como trazadora de correlación mundial con Indonesia (Cuenca del Circumpacifico) para contribuir al mejor conocimiento del Grupo Yura y su incorporación dentro de la zonación estratigráfica para el Titoniano, edad de mucho interés en la formación de recursos fósiles en el mundo.

\section{LOCALIZACIÓN Y GEOLOGÍA REGIONAL.}

Se llega al sitio fosilífero transitando por la carretera Puno - Tiquillaca Km 11. Saliendo de esta última ciudad con rumbo hacia el Sur, a pocos kilómetros de recorrido, se toma la desviación al Cerro Yanaorco. Los afloramientos forman parte de un anticlinal (rumbo del eje S75E), en el cual se encuentran los ejemplares estudiados. Sobre tal trayecto se han medido la columna estratigráfica, con el afloramiento ubicado con GPS 370211 y 247373, a un costado del camino (Figura 1). 
Figura 1. Afloramiento del Cerro Yana Apacheta

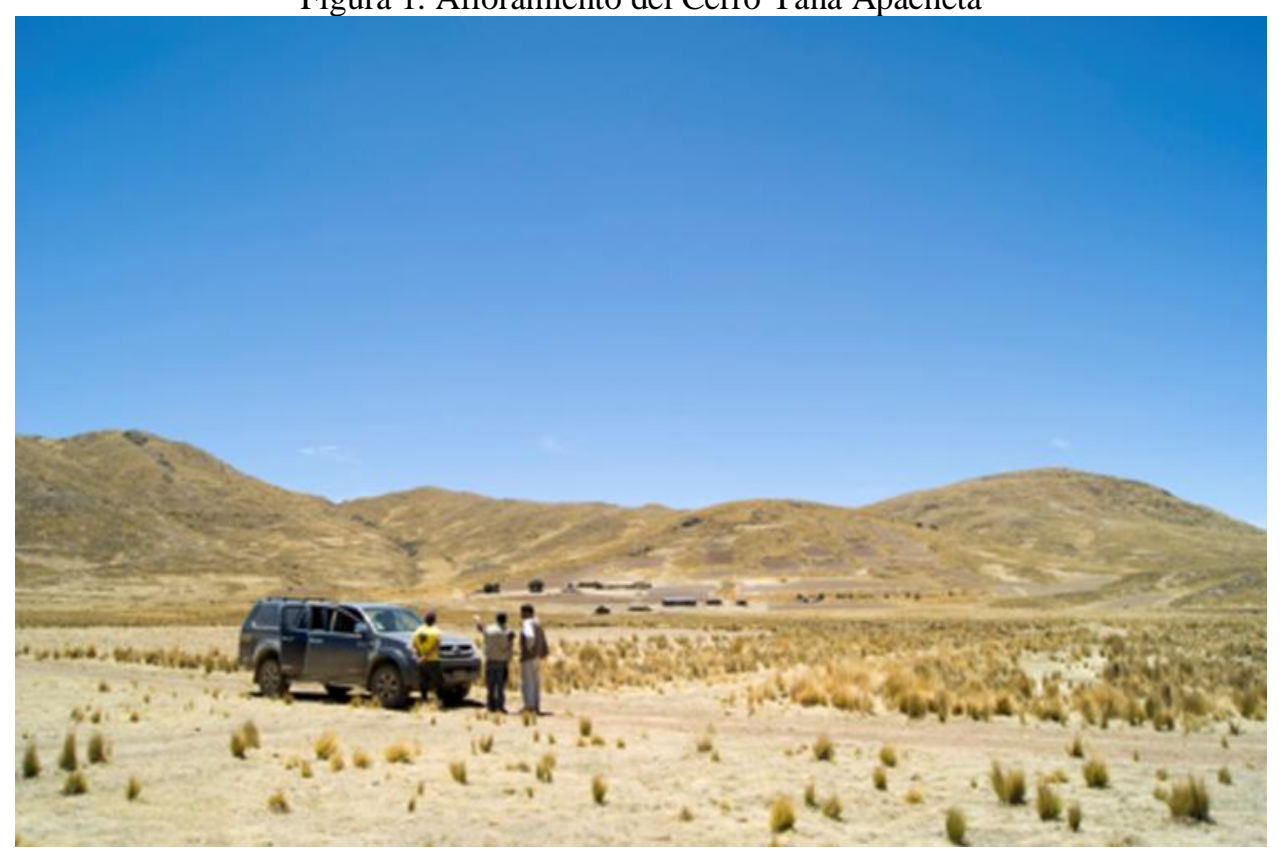

En la Cuenca mesozoica de Arequipa las rocas volcánicas del arco Jurásico Chocolate (Jenks, 1948; Martinez \& Cervantes, 2003), infrayacen a sucesiones detríticas y carbonatadas depositadas durante el Jurásico Inferior a medio. Estas unidades han sido denominadas en Arequipa como las formaciones Chocolate y Socosani. Sobre estas, se describe al Grupo Yura (formaciones: Puente, Cachíos, Labra y Gramadal) (Jenks, 1948; Benavides, 1962; Vicente, 1981). Mientras que en Puno, se ha considerado al Grupo Lagunillas (Jaillard \& Santander, 1992), como las unidades correlacionables con el Grupo Yura (específicamente a las formaciones Puente y Cachios). De acuerdo a Vicente (1981) y con las observaciones aún inéditas del Proyecto GR21, la información obtenida en la zona de Tiquillaca, tanto estratigráfica como estructuralmente, tienen una gran similitud con la geología de la zona de Yura, donde las columnas estratigráficas levantadas en el Cerro Yanacoto y la Quebrada de El Burro en Yura están conformadas por conglomerados y areniscas. Este material detrítico, es el producto de la erosión de importantes y potentes zonas volcánicas (Arco Jurásico Chocolate). 
Figura 2. Mapa de Ubicación de la zona estudiada

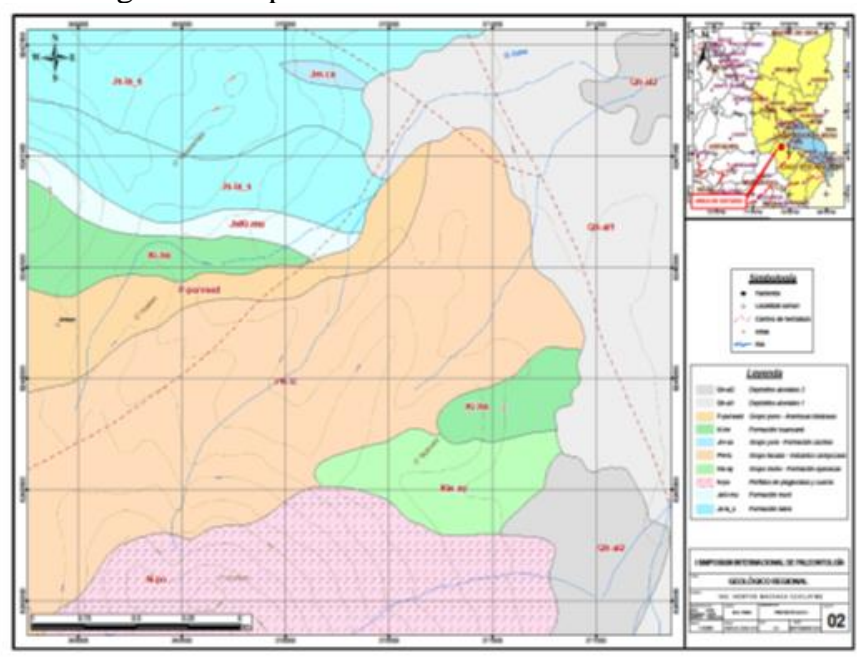

\section{METODOLOGÍA}

Se inspeccionó el afloramiento en busca de fósiles posteriormente al periodo de avenidas, lo que facilitó la recolecta de amonites mayores a 5 centímetros, así como fragmentos que se reconstruyeron con soporte de yeso. El muestreo de fósiles y litología macroscópica se realizó conjuntamente con el levantamiento del perfil local del cerro Yana Apacheta. Los amonoideos fueron descritos inicialmente por el número de vueltas, diámetro máximo, altura de la vuelta externa y su proporción respecto a la segunda vuelta, así como la tasa de enrollamiento compuesta por los parámetros W, D. S.

\section{RESULTADOS}

Cinco ejemplares de amonoides contenidos en sendas concreciones calcáreas con limolita arenosa; determinados como Himalayites uhlig in BOEHM (1904) con sinonimia en la especie Himalayite truebi DOUVILLE (1912). Indican el Tithoniano Superior. Tal determinación se otorga con las siguientes bases: Concha evoluta con vueltas interiores ligeramente comprimidas y vuelta externa ligeramente redondeada en sección transversal. El Costillaje de las vueltas internas es biplicado y simple. Sobre las vueltas medias y externas hay tubérculos esporádicos meso-laterales (espinas) desde las que ramifican 2 a 4 costillas secundarias. Hay costillas primarias intermedias entre las anteriores que permanecen simples y hay costillas secundarias alternas entre las primarias. El vientre muestra un surco medio o una franja plana lisa, que se difumina sobre la última vuelta. Los ejemplares se apegan casi a todo con respecto a la diagnosis anterior, excepto que las costillas biplicadas de las vueltas internas casi no son visibles. Adicionalmente los parámetros W (tasa de expansión de la vuelta), D (distancia de apertura del eje de enrollamiento) y, S (forma de la apertura definida por el eje del enrollamiento) que describen la proporción y tasa de enrollamiento son de $1.46,0.45$ y 0.61 respectivamente, lo que corresponde a enrollamiento semi-evoluto basado en las medidas: $a=20.97 \mathrm{~mm}$ de altura de la vuelta; $b=12.88 \mathrm{~mm}$ de 
espesor de la vuelta; $\mathrm{c}=17.00 \mathrm{~mm}$ de radio umbilical mayor; $\mathrm{d}=37.61 \mathrm{~mm}$ de radio mayor; $\mathrm{e}=31.04 \mathrm{~mm}$ de radio menor. Esta determinación hace sinonimia con Himalayites treubi Böhem de la figura 4 a y b de Westermann (2000) placa 86, misma publicada en Arkell (1956) pero que se menciona como Himalayites negherburghi BÖHEM (1904).

\section{DISCUSIÓN}

El ejemplar H. treubi sujiere una correlación regional con la Formación Labra en Arequipa y con la Formación Chachacumane en Tacna. La correlación de Puno con estas dos localidades, aclara la división con la Formación Cachios. Aunque están consideradas en la figura como pertenecientes al grupo Yura, queda evidente que es una asignación en desuso y hay que señalar que la división bioestratigráfica con la edad Titoniana, marca diferencia y que los estratos fosilíferos dejen de ser grupo Yura, si es que la transición estratigrafíca/petrológica así lo permiten desde un punto de vista ambiental sedimentario; pero si la litología da indicios de transición sedimentaria gradual o de continuidad entonces podrían ser indicios de que la división (zonación estándar) con el Tithoniano pudiera reconsiderarse localmente. Por otra parte la correlación con el Tithoniano Superior de Indonesia traza correlación cronológica con el Jurásico del Circumpacifico.

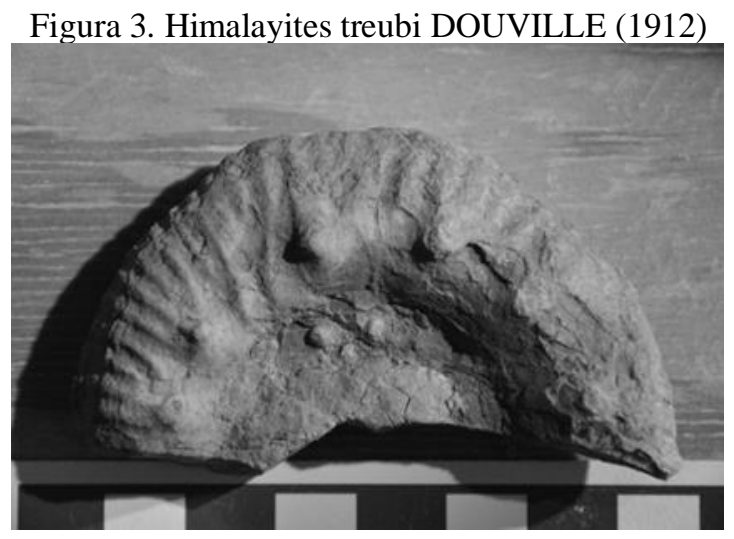

Por otra parte los pulsos de transgresión/regresión de la parte final del jurásico vistas en la figura 4 deberá confirmarse la litología de esta localidad, los fósiles al parecer están en de arenisca limosa y carbonatada, asociable a fondos someros en un contexto de regresión, como los ilustrados en la ultima fase del Kimeridgiano de la Fm. Cachios (Figura 4): Esto podría interpretarse como la parte final del Jurásico que dio lugar a grandes cambios de terrestralización definitiva de ese sector del continente sudamericano; Es decir la que secuencia de Yana Apacheta y su formación equivalente "Labra" podrían representar la transición final hacia el Cretácico. De esta manera el trabajo pionero de Aldo Alfonso Alván de la Cruz (2009) sobre la descripción de Jurásico Medio e inferior resulta complementado. 


\section{SOUTH FLORIDA}

JOURNAL OF DEVELOPMENT

Figura 4. Columna estratigráfica generalizada de los estratos jurásicos de la Cuenca Arequipa (tomado de Wilson \& García, 1962; Vicente, 1981, y de los trabajos del INGEMMET, 2007, 2008, 2009, 2013), donde aún no se han registrado sedimentos tithonianos.

\begin{tabular}{|c|c|c|c|c|c|}
\hline Sist. & Serie & Piso & Arequipa & Litologia & Tacna \\
\hline & \multirow{4}{*}{ Superior } & Titoniano & \multirow[b]{2}{*}{$\begin{array}{ll}\cong & \text { Fm. Cachios }\end{array}$} & & Fm. Chachacumane \\
\hline & & Kimmeridgiano & & & \multirow{3}{*}{$\begin{array}{c}\mathrm{Fm} . \\
\text { Ataspaca }\end{array}$} \\
\hline & & Oxfordiano & \multirow{2}{*}{ Fm. Puente } & & \\
\hline & & Caloviano & & & \\
\hline \multirow{5}{*}{$\begin{array}{l}\frac{0}{0} \\
\frac{0}{0} \\
\frac{\pi}{3} \\
\frac{10}{3}\end{array}$} & \multirow{3}{*}{ Medio } & Batoniano & \multirow{4}{*}{ Fm. Socosani } & & \multirow{4}{*}{$\begin{array}{c}\mathrm{Fm} . \\
\text { San Francisco }\end{array}$} \\
\hline & & Bajociano & & & \\
\hline & & Aaleniano & & & \\
\hline & \multirow[b]{2}{*}{ Infaringr } & Toarciano & & & \\
\hline & & Pliensbachiano & \multirow{6}{*}{ Fm. Chocolate } & 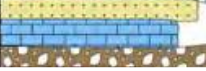 & \multirow[t]{2}{*}{ Fm. Pelado } \\
\hline & & Sinemuriano & & & \\
\hline & & Hettangiano & & & \multirow{4}{*}{ Fm. Junerata } \\
\hline O & \multirow{3}{*}{ Superior } & Raetiano & & & \\
\hline$\frac{\pi}{\pi}$ & & Noriano & & & \\
\hline$\vdash$ & & Carniano & & & \\
\hline
\end{tabular}




\section{REFERENCIAS}

Alván, A. \& Acosta, A. (2009).- Sedimentary facies and ammonites relations between Arequipa and Tacna during Lower to Middle Jurassic. 21st Lateinamerika-Kolloquium LAK 2009, Abstracts; p. 72-77.

Arkell, WM Furnish, Bernhard Kummel, AK Miller, RC Moore, OH Schindewolf, PC Sylvester-Bradley y CW Wright. (1957). - Tratado de paleontología de invertebrados, parte 1, molusca 4, Raymond C. Moore. 490 pp., 558 fig.

León, I. (1981).- Antecedentes sedimentológicos del Jurásico-Cretácico inferior en la zona de Yura. Tesis de Bachiller, Universidad Nacional de San Agustín; 100 p.

Palacios, O., De la Cruz. N., Klink, B.A., Ellison, R.A., Hawkins, M.P. (1991), Geología de la Cordillera Occidental y Altiplano al Oeste del Lago Titicaca - Sur del Perú (Proyecto Integrado del Sur). Serie A: Carta Geológica Nacional., Dirección de Geología Regional, INGEMMET, Lima, Perú., Boletín N 42, $257 \mathrm{p}$.

Pino, A., Semperé, T., Jacay, J. \& Fornari, M. (2004).- Estratigrafía, paleogeografía y paleotectónica del intervalo Paleozoico superior-Cretáceo inferior en el área de Mal Paso-Palca (Tacna).Publicación Especial № 5, Sociedad Geológica del Perú; p. 15-44.

Sandoval, J., O’Dogherty, L., Vera, J. \& Guex, J. (2002).- Sea-level changes and ammonite faunal turnover during the Lias/Dogger transition in the western Thetys. Bull. Soc. Geol. France, Tomo 173, $\mathrm{N}^{\circ}$ $1 ;$ p. 57-66.

Vargas, L. (1970).- Geología del cuadrángulo de Arequipa (Hoja 33-s). Servicio de Geología yMinería de Perú actualmente INGEMMET), Serie A: Carta Geológica Nacional, N² 24; 64 p.

Westermann, G., Riccardi A., Palacios O. \& Rangel C. (1980).- Jurásico medio en el Perú. Boletín Nº9, Instituto Geológico Minero y Metalúrgico; 63 p.

Westermann, G. (1980).- Ammonite Biochronology and Biogeography of the Circum-Pacific MiddleJurassic. Systematics Association, Special Volume N 18; p. 459-498. 